\title{
Major pulmonary embolism, a correlation of clinical findings, haemodynamics, pulmonary angiography, and pathological physiology
}

\author{
I. G. McDonald, J. Hirsh, G. S. Hale, and E. F. O'Sullivan \\ From the Cardiovascular Unit and the University of Melbourne Department of Medicine, \\ St. Vincent's Hospital, Melbourne, Victoria, Australia
}

Twenty-three patients with major pulmonary embolism were examined clinically and investigated by cardiac catheterization with selective pulmonary angiography. Two-thirds of the patients had clinical evidence of acute right ventricular strain. The electrocardiogram was considered 'typical' of acute right heart strain in 39 per cent and the chest $\mathrm{x}$-ray showed changes considered or suggestive of pulmonary embolism in 83 per cent. Important atypical clinical features included the absence of dyspnoea in one patient, absence of elevation of the jugular venous pressure in 2 patients, and conspicuous pulsus paradoxus in one patient. Wheezing was never seen. The pulmonary angiogram revealed saddle embolism in 13 per cent, severe occlusion of the right or left pulmonary artery in 61 per cent, and obstruction confined to lobar and more distal arteries in 26 per cent. The severity of pulmonary vascular obstruction was best indicated, not by the severity of pulmonary hypertension which was moderate, but by the severity of depression of the stroke volume and cardiac output.

During the same period, 26 patients were referred as possible pulmonary embolism cases but were found to have another disease as the cause of the symptoms and signs. Pneumonia, pulmonary collapse, left ventricular failure, acute cardiac tamponade, and Gram-negative septicaemia were the conditions which most closely resembled major pulmonary embolism in their clinical presentation.

Pulmonary embolism remains a common cause of death even in the young and previously healthy. This high mortality could undoubtedly be reduced by adequate prophylaxis of high risk patients and adequate treatment of patients with minor thromboembolic episodes (Thomas, 1965; Barritt and Jordan, 1960; Wechsler et al., 1969; Little and Loewenthal, 1968). It is likely also that the mortality could be further reduced by treatment of selected patients with acute major pulmonary embolism with pulmonary embolectomy (Cross and Mowlem, 1967; Cooley and Beall, 1968) or thrombolytic therapy (Hirsh et al., 1968; Walsh, Stengle, and Sherry, 1969). The feasibility of these forms of therapy depends on early and accurate diagnosis. Diagnostic precision has been greatly improved by the availability of pulmonary angiography (Simon and Sasahara, I965) and radio-isotopic lung scanning (Tow

Received 7 June 1971. and Wagner, 1967). The availability of more accurate diagnostic methods and of more specific treatment has heightened the responsibility of the attending clinician to diagnose correctly or at least to suspect the diagnosis of major pulmonary embolism. Furthermore, the interpretation of these investigations and the selection of the most appropriate form of therapy require greater understanding of the pathological physiology of major pulmonary embolism. During a recent prospective study of the rate of natural resolution of major pulmonary embolism and of the effects of streptokinase therapy (Hirsh et al., 1968; McDonald, Hirsh, and Hale, 1971), an opportunity was provided to correlate the clinical findings with haemodynamic and angiographic investigations and to analyse these data in the light of what is currently known about the physiology of major pulmonary embolism in man. This study also highlighted certain atypical features and diagnostic difficulties which will be discussed. 


\section{Methods}

Selection of patients The criteria for selection were severe pulmonary embolism demonstrated by pulmonary angiography and pulmonary hypertension (mean pulmonary arterial pressure $>$ I5 $\mathrm{mmHg}$ ). Of the 23 patients studied, I I were men and 12 women and their average age was 49.2 years (range 23 to 73 years). Associated cardiac or pulmonary disease was present in 4 patients. Ischaemic heart disease was present in 2 patients; one had been asymptomatic and the other had pulmonary venous congestion. One patient had mitral regurgitation but no evidence of pulmonary congestion and the remaining one had recurrent bronchitis but a normal exercise tolerance. The time lapse between the most recent clinical episode of major pulmonary embolism and of presentation was less than 2 hours in 5 patients, between 2 and 12 hours in 9 patients, between 12 and 48 hours in 4 patients, and more than 48 hours in 3 patients. Symptoms were progressive in 2 patients, so that the onset of major pulmonary embolism could not be accurately determined. The commonest factor predisposing to pulmonary embolism was surgery $(52 \%)$ and other important associations were varicose veins $(22 \%)$, prolonged immobility ( $17 \%$ ), and neoplasia ( $17 \%$ ). In 35 per cent of the patients, there was clinical evidence of pulmonary embolism during the preceding 4 weeks and in 17 per cent evidence of embolism more than 4 weeks previously.

Analysis of chest x-ray and electrocardiograms In order to assess the contribution of these investigations to the diagnosis of major pulmonary embolism, both the chest $x$-ray and electrocardiogram were classified as being either 'normal', 'typical' of pulmonary embolism, 'suggestive' of pulmonary embolism, or as showing 'non-specific' changes. The chest $x$-ray was regarded as 'typical' only if it showed a definite localized area of pulmonary oligaemia (Westermark, 1938). Localized pulmonary plethora was also detected in some patients and was a useful confirmatory finding when localized oligaemia had been suspected, but was not considered to be a sufficiently unequivocal finding as an isolated sign. Changes 'suggestive' of pulmonary embolism included focal shadows consistent with recent pulmonary infarction, localized soft shadows suggesting pulmonary oedema, evidence of reduction in the volume of one or both lungs (particularly involving the lower lobes with rise of the diaphragm), and changes in the size and shape of the heart suggestive of enlargement of the right ventricle, of the pulmonary arteries, or of the superior vena cava. All other changes were regarded as 'non-specific'. The electrocardiogram was classified in a similar manner according to previously established criteria (Oram and Davies, I967).

Right heart and brachial or radial arterial catheterization were performed immediately after the initial clinical assessment. Brachial arterial (BA), pulmonary arterial (PA), and right atrial pressures (RA) were measured with reference to a point $5 \mathrm{~cm}$ posterior to the sternal angle. Car- diac output (CO) was measured by the indicator dilution technique. The index, total pulmonary resistance $\left(\frac{\overline{\mathrm{PA}}}{\mathrm{CO}}\right)$, was used to assess changes in pulmonary vascular resistance and the systemic vascular resistance, $\left(\frac{\overline{\mathrm{BA}}-\overline{\mathrm{RA}}}{\mathrm{CO}}\right)$ was also calculated. After the haemodynamic measurements, pulmonary angiography was performed in the anteroposterior projection using a rapid film changer (Elema-Schonander). The degree of pulmonary vascular occlusion was independently estimated from the pulmonary angiogram by 3 observers and the average value of their estimates was recorded. Other investigations performed in these patients have been reported elsewhere and included measurements of the haemodynamic response to a continuous infusion of isoprenaline (McDonald et al., I968), tests of pulmonary function (Hale et al., 1968), and assessment of the relative effects of heparin and streptokinase on the rate of resolution of pulmonary embolism (Hirsh et al., I968).

Treatment All patients were initially examined in consultation with the cardiothoracic surgical team and, after investigation, were nursed in a coronary care unit with continuous monitoring of the electrocardiogram. All received intravenous heparin and digitalis but the infusion of isoprenaline was continued only when clinically indicated. Streptokinase and heparin were administered as previously described (Hirsh et al., I968). Pulmonary embolectomy on cardiopulmonary bypass was successfully undertaken in 2 of these patients and an emergency Trendelenburg operation was unsuccessfully attempted in another. Ligation of the inferior vena cava was performed in I patient. Seven patients died. Pulmonary embolism was considered to be the major cause of death in 3 patients and to have been a contributing factor in a further 3 patients. One patient was killed in a road accident.

\section{Results}

Clinical features - symptoms (Table I) Dyspnoea was the commonest symptom and was severe in 17 of the 23 patients, moderately severe in 2 , and mild in 3 patients. Of particular interest was the patient who did

TABLE I Symptoms

\begin{tabular}{lrr}
\hline & Per cent & No. \\
\hline Dyspnoea & 96 & 22 \\
Neurological & 61 & I4 \\
Chest pain & & \\
'Cardiac' & I3 & 3 \\
Pleuritic & 9 & 2 \\
Both & 4 & I \\
\hline
\end{tabular}


TABLE 2 Clinical signs

\begin{tabular}{lrr}
\hline & Per cent & No. \\
\hline $\begin{array}{lr}\text { Cardiovascular } \\
\text { Rise of jugular venous pressure }\end{array}$ & 91 & 21 \\
$\begin{array}{l}\text { Right ventricular lift or gallop } \\
\text { rhythm }\end{array}$ & 61 & 14 \\
Electrocardiogram typical of acute & & \\
$\quad$ right ventricular strain & 39 & 9 \\
$\begin{array}{l}\text { Pulsus paradoxus } \\
\text { Clinical shock }\end{array}$ & 4 & I \\
Respiratory & 13 & 3 \\
$\quad$ Tachypnoea & & \\
Cyanosis & 100 & 23 \\
$\quad$ Unilateral restriction of chest wall & 61 & 14 \\
$\quad$ movement & 26 & 6 \\
$\quad$ Local lung signs & 78 & 18 \\
Fever $\left(>3^{\circ}\right)$ & 35 & 8 \\
\hline
\end{tabular}

not have dyspnoea. She was a woman, aged 32 years, in whom pulmonary angiography had demonstrated a saddle embolus. Her clinical course was unusual in that it was punctuated by recurrent syncope and 2 episodes of cardiac arrest. In the remaining patients, neurological symptoms were transient and occurred immediately after embolism in the form of syncope ( $35 \%$ ) or transient dizziness $(26 \%)$. Chest pain suggestive of myocardial ischaemia was relatively uncommon (13\%), and when present occurred early and quickly subsided. Pleuritic chest pain was a complaint on presentation in 9 per cent of the patients and both cardiac and pleuritic pain were present in I patient.

Clinical features - signs (Table 2) The jugular venous pressure was raised in $2 \mathrm{I}$ patients $(91 \%)$. The patient with saddle embolism already discussed was one of the exceptions, and the other was a young woman with severe pulmonary vascular obstruction whose clinical presentation was otherwise not unusual. Other signs of acute right heart strain and of pulmonary hypertension were usually detectable. In 6I per cent of patients, a right ventricular parasternal lift or definite right ventricular gallop rhythm or both were present. The electrocardiogram was classified as 'typical' of pulmonary embolism in 39 per cent of the patients and 'suggestive' in a further 39 per cent. One of these 3 manifestations of acute right heart strain was definitely present in approximately two-thirds of our patients, and at least one of these signs was suspected in more than half of the remaining patients.

The clinical diagnosis of shock was based on conventional criteria - systolic hypotension (systolic blood pressure < $100 \mathrm{mmHg}$ ), cool and sweaty skin, and oliguria (urinary output $<30 \mathrm{ml} /$ hour). Judged by these criteria, 3 patients were considered to be in shock at the time of cardiac catheterization and pulmonary angiography.

Pulsus paradoxus was detected clinically in one patient who was not in shock. The second heart sound was not found helpful in diagnosis.

The most important of the respiratory signs was tachypnoea which was invariably present and usually severe. Unilateral restriction of chest wall movements was also common ( $26 \%$ ) and the breath sounds were frequently diminished over large areas of the chest, usually in association with moist adventitious sounds. It is noteworthy that wheezing was not observed in any of these patients.

A fever of $38^{\circ} \mathrm{C}$ or more was not unusual and was a potentially confusing sign. Its cause could not be defined with certainty in individual patients, but its presence could usually be explained by pulmonary infarction, deep venous thrombosis, or an associated infection.

The chest $\mathrm{x}$-ray (Table 3 ) showed a definite area of oligaemia and was therefore considered 'typical' of pulmonary embolism in ro patients $(44 \%)$. In 9 patients $(39 \%)$ the changes were considered 'suggestive' of pulmonary embolism and 'non-specific' in 2 patients $(9 \%)$. The lung fields were considered to be within normal limits in only 2 patients $(9 \%)$.

Differential diagnosis During this study, there were 26 patients in whom other diseases had simulated pulmonary embolism sufficiently closely to lead to their referral for consideration of pulmonary angiography. The diseases which caused such diagnostic difficulty has been classified in Table 4 as cardiac, respiratory, and non-cardiorespiratory. This Table indicates that most difficulty was encountered with acute respiratory diseases, pulmonary venous congestion, acute cardiac tamponade, and Gram-negative septicaemia.

TABLE 3 Chest $\mathrm{x}$-ray

\begin{tabular}{lrr}
\hline & Per cent & No. \\
\hline Typical & 44 & 10 \\
Suggestive & 39 & 9 \\
Non-specific & 9 & 2 \\
Normal lung fields & 9 & 2
\end{tabular}

See text for classification. 


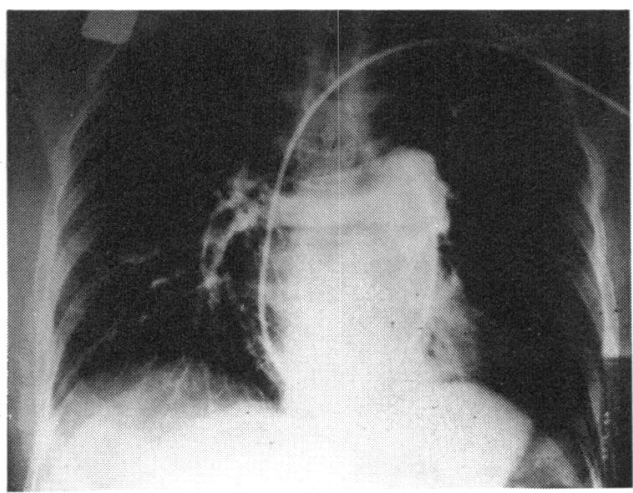

FIG. I Angiogram, showing saddle pulmonary embolism.

Pulmonary angiography (Table 5, Fig. I-5) The average degree of pulmonary vascular obstruction was 53.4 per cent (range 25 to $75 \%$ ). In 20 of the 23 patients the degree of occlusion exceeded 45 per cent and in 7 of them exceeded 65 per cent. The angiograms were classified according to the site of the most proximal filling defect (Table 5). In no patient was an embolus visible in the main pulmonary artery but in 3 patients (13\%) saddle embolism was demonstrated (Fig. I). In 14 patients $(6 \mathrm{r} \%)$, filling defects were visible in the right or left branches of the pulmonary artery or in both branches. Included were 4 patients ( $17 \%$ ) with total or subtotal occlusion of I main branch with partial occlusion of the other (Fig. 2), 5 patients ( $22 \%$ ) with partial occlusion of both main pulmonary arteries

FIG. 2 Angiogram showing subtotal occlusion of the left pulmonary artery with partial

occlusion of the right pulmonary artery.
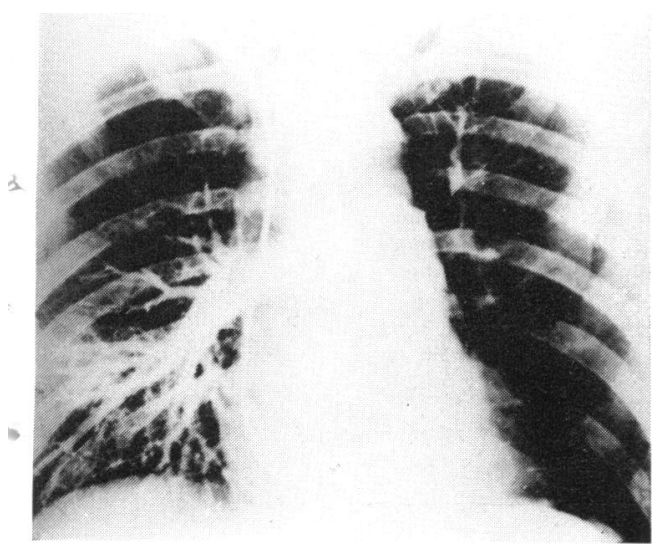

\section{TABLE 4 Differential diagnosis}

a) Cardiac disease

Pulmonary congestion: myocardial ischaemia or infarction alcoholic cardiomyopathy intravenous fluid overload

Cardiac rupture, myocardial infarction

Dissecting aneurysm of left coronary artery

Cardiac tamponade: dissecting aneurysm

postoperative haemopericardium

recurrent pericarditis with effusion

Total

b) Respiratory disease

\begin{tabular}{ll} 
Emphysema, chronic bronchitis & 2 \\
Pneumonia or pulmonary collapse & 6 \\
\hline Total & 8 \\
\hline
\end{tabular}

c) Non-cardiorespiratory

Gram-negative septicaemia

Acute renal failure, gastroenteritis, post partum

Retroperitoneal haemorrhage, postoperative

Mesenteric embolism, severe acidosis

Total

(Fig. 3), and 5 patients (22\%) with partial occlusion of one main branch of the pulmonary artery with occlusion of multiple lobar vessels on the opposite side (Fig. 4). When the right or left pulmonary artery was partially occluded, the typical appearance was of a large embolus or mass of emboli which occupied the terminal portion of the artery and extended into and obstructed variable number of its lobar branches. In the remaining 6 patients (26\%), filling defects were not visible in the right or left pulmonary arteries so that obstruction seemed to be confined to lobar and more distal arteries (Fig. 5).

Haemodynamics (Table 6) The results of the haemodynamic measurements have been presented as average values for 2 groups of patients. The first group comprised the 3 patients who were considered to be in shock on clinical grounds at the time of cardiac catheterization, and the second group the 20 patients who were not considered to be in shock. In those patients who were not shocked, the total pulmonary resistance was approximately twice the upper limit of normal (3 units), and the pulmonary arterial and right atrial pressures were moderately raised. The reduction in stroke volume was quite severe (normal value $>35 \mathrm{ml} / \mathrm{m}^{2}$ ) but the sinus tachycardia partially compensated for the reduced stroke volume so that the cardiac output was at the lower limits of normal (normal 
TABLE 5 Pulmonary angiography

\begin{tabular}{llc}
\hline & Per cent & No. \\
\hline Saddle embolism & 13 & 3 \\
$\begin{array}{l}\text { Total or subtotal occlusion of one } \\
\quad \text { main branch }\end{array}$ & 17 & 4 \\
$\begin{array}{l}\text { Partial occlusion of both main branches } \\
\text { Partial occlusion of one main branch }\end{array}$ & 22 & 5 \\
$\begin{array}{l}\text { Occlusion confined to lobar and distal } \\
\text { arteries }\end{array}$ & 26 & 5 \\
\hline
\end{tabular}

value $\left.>2.51 . / \mathrm{m}^{2}\right)$. The systemic vascular resistance was at the upper limit of normal (20 units). In the shocked patients, the total pulmonary resistance was higher, the reduction in stroke volume more severe, the tachycardia of similar magnitude, the cardiac output was lower, and the systemic vascular resistance higher than those who were not shocked. In contrast, the severity of pulmonary hypertension was similar in both shocked and nonshocked patients and the right atrial pressure was only slightly higher in the shocked group.

Though pulsus paradoxus was detected clinically in one patient only, exaggeration of the inspiratory fluctuation of systemic arterial blood pressure was common, a finding which will be presented in detail elsewhere.

\section{Discussion}

Problems of case selection Clinical studies of major pulmonary embolism are often difficult to compare with one another because of variations in the average severity of pulmonary embolism and in the incidence of complicating cardiopulmonary disease. Variations in the severity of pulmonary embolism may be subtly introduced by differences in the time lapse between embolism and initial examination, since the mortality of major pulmonary embolism is high during the first few

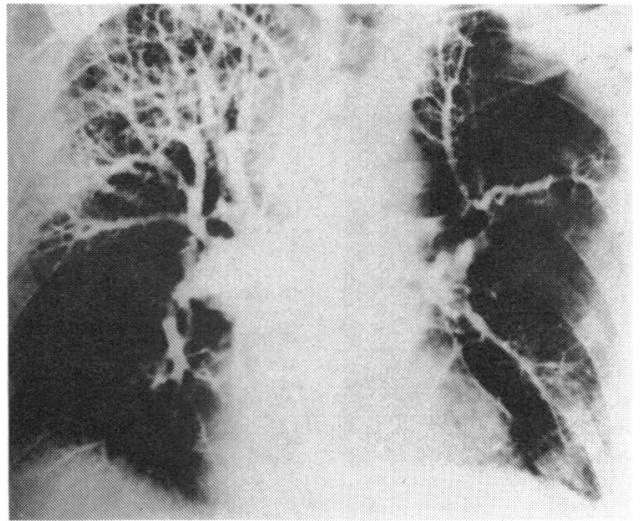

F IG. 3 Angiogram showing partial occlusion of the termination of both right and left pulmonary arteries.

hours, but rapidly declines (Soloff and Rodman, 1967). This is the probable explanation of the low mortality in our series, since all of the patients were initially examined some hours or days after the recent episode of major pulmonary embolism. In addition, there was an understandable tendency for younger and otherwise healthy patients to be referred for pulmonary angiography rather than older patients and those with advanced associated disease. This tendency is probably also reflected in the high proportion of postoperative patients in our study and in the low incidence of complicating cardiopulmonary disease. Despite such influences, our patients are representative of those referred for investigation of suspected major pulmonary embolism in a large general hospital. A similar group of patients was studied by Miller and Sutton (1970). The clinical manifestations and haemodynamic changes closely resemble our own.

TABLE 6 Haemodynamics

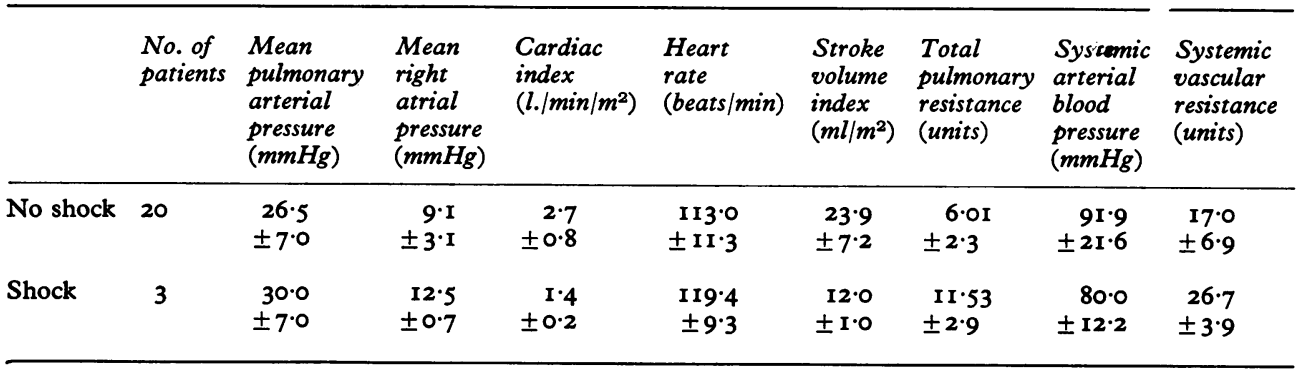

$\pm=$ I SD. 


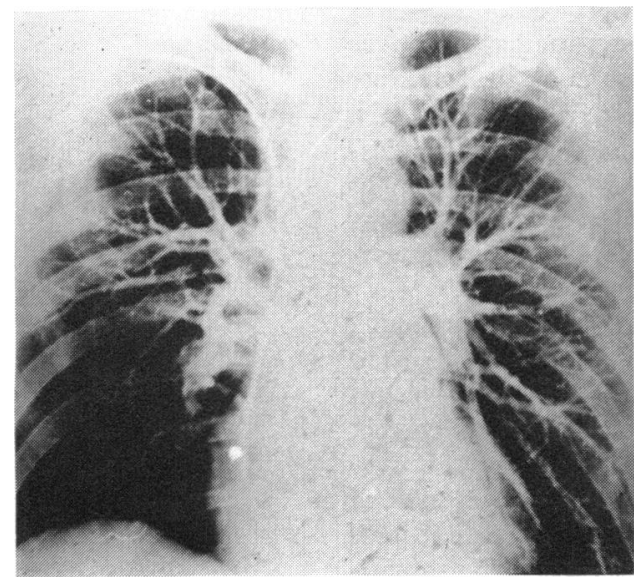

FI G. 4 Angiogram showing partial occlusion of the left pulmonary artery with filling defects extending into the lobar branches and occlusion of lobar and segmental arteries (especially lingular and lower lobe) on the left side.

Clinical features The typical manifestations of major pulmonary embolism encountered in this study were dyspnoea, cyanosis, rise of the jugular venous pressure and other evidence of acute right heart strain, nonspecific chest physical signs, and, frequently, inconspicuous changes in the chest $x$-ray. These clinical findings are well known (Dexter et al., 1960; Marshall, 1965; Gray, 1966), and it is our intention to concentrate on the diagnostic difficulties encountered. The following

FIG. 5 Multiple filling defects, especially in lower lobe arteries bilaterally, with obstruction to blood flow.

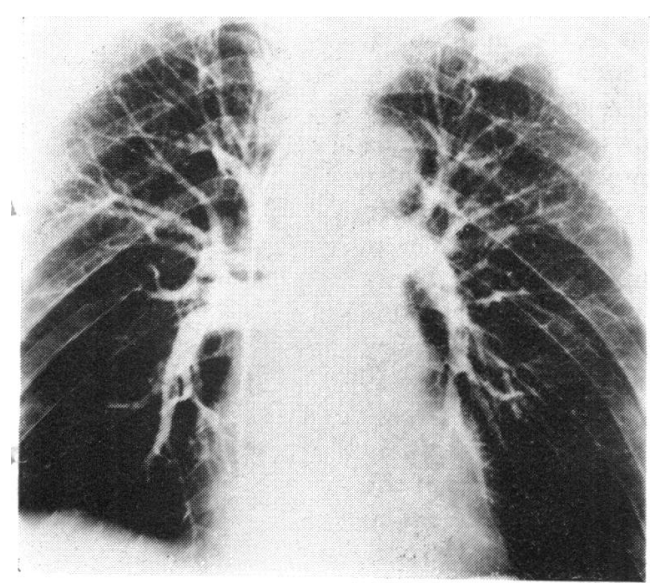

classification of diagnostic problems in major pulmonary embolism will be used as a basis for discussion. (a) An atypical mode of presentation of major pulmonary embolism with either the absence of the classical features of dyspnoea or of the rise of the jugular venous pressure, or prominence of unusual features such as chest pain, neurological symptoms, pulsus paradoxus, wheezing, or fever. (b) Simulation of major pulmonary embolism by other diseases which caused various combinations of sudden dyspnoea, circulatory collapse, or chest pain.

Atypical presentation of pulmonary embolism Diagnostic difficulty due to absence of dyspnoea was encountered in only one patient. In this patient the jugular venous pressure was not raised at the time of the initial examination and the absence of both of these features was believed to be due to the intermittent nature of severe pulmonary vascular obstruction (McDonald et al. 1970). There was one other patient in whom the jugular venous pressure was not raised but in this case it seemed more likely that this was due to the subacute nature of the onset of pulmonary embolic obstruction.

Clinical improvement during the initial 24 hours after investigation was the rule even in patients who did not receive thrombolytic therapy. The jugular venous pressure usually fell, a right ventricular lift tended to regress, and a gallop rhythm to become softer or inaudible. An important corollary is that signs of acute right ventricular failure may be absent or inconspicuous in patients seen more than 24 hours after major embolism, a finding emphasized by Miller and Sutton (1970). A physical sign which created some diagnostic doubt was pulsus paradoxus (Burdine and Wallace, 1965). This is recognized as a classical sign of cardiac tamponade, and the clinical presentation of acute cardiac tamponade due to haemorrhage may closely resemble that of major pulmonary embolism. Though we found clinically obvious pulsus paradoxus to be uncommon in major pulmonary embolism, exaggeration of the normal respiratory fluctuation in systemic arterial blood pressure was detected frequently at cardiac catheterization. Of the other potentially confusing manifestations which have been described in major pulmonary embolism, neither chest pain suggestive of myocardial ischaemia nor neurological symptoms caused any diagnostic difficulty, while fever was no longer considered atypical once its high incidence had been appreciated. Several authors have described wheezing in association with clinical pulmon- 
ary embolism (Gurewich, Sasahara, and Stein, 1965; Olazábal et al., 1968; Webster et al., I966) and bronchoconstriction has been produced by experimental pulmonary embolism in dogs (Gurewich et al., 1965). However, we did not observe wheezing in any of our patients and this was also the experience in another large series of patients with major pulmonary embolism (Sutton, Honey, and Gibson, 1969). Wheezing has been described as part of a clinical syndrome of chest pain, peripheral venous thrombosis, and dyspnoea (Gurewich et al., 1965) and also when pulmonary embolism occurs in patients who are prone to bronchial asthma (Olazábal et al., I968). Wheezing may also be present when pulmonary embolism complicates preexisting pulmonary venous congestion or in the rare event of pulmonary oedema precipitated by pulmonary embolism (Luisada and Cardi, 1956). Nevertheless, wheezing as a manifestation of acute major pulmonary embolism seems to be uncommon.

Simulation of pulmonary embolism The illnesses that were most difficult to distinguish from major pulmonary embolism were pneumonia or pulmonary collapse, acute pulmonary venous congestion, acute cardiac tamponade, and Gram-negative septicaemia. The differentiation of acute respiratory disease from major pulmonary embolism is a common clinical problem. The important distinguishing features were a more gradual onset of dyspnoea often accompanied by higher fever and constitutional symptoms, and a chest $x$-ray which usually showed an opacity of sufficient extent to account for severity of both dyspnoea and hypoxia as well as for the chest physical signs. The finding of hypercapnia on blood gas analysis also favoured pulmonary infection. The pulmonary angiogram sometimes showed a reduction of blood flow through a consolidated or collapsed segment of lung which sometimes bore a superficial resemblance to the effects of pulmonary embolism, but the important distinction was the absence of filling defects.

Diagnostic difficulty was also experienced in patients with pulmonary venous congestion in whom signs of pulmonary hypertension were prominent. Though the presence of orthopnoea, paroxysmal nocturnal dyspnoea, wheezing, and the characteristic radiological appearance were usually sufficient to indicate that pulmonary venous congestion was the likely cause of dyspnoea, it was often impossible to exclude with confidence associated pulmonary embolism to which such patients are predisposed. For this reason, these patients were treated with heparin when the diagnosis of pulmonary embolism was suspected, and pulmonary angiography was avoided because of its tendency to aggravate pulmonary venous congestion. There were 2 patients in whom pulmonary venous congestion was due to excessive administration of intravenous fluid postoperatively and it was the fluid balance chart which provided evidence suggestive of this diagnosis.

In 3 patients with acute cardiac tamponade, the clinical presentation initially suggested major pulmonary embolism but the important clinical evidence that this was not the correct diagnosis was the relative mildness of dyspnoea and tachypnoea and the presence of some pericardial pain. In a third patient with acute cardiac tamponade, right atrial angiography was required to confirm the diagnosis and pulmonary angiography to exclude pulmonary embolism with confidence.

Gram-negative septicaemia with systemic hypotension and clinical signs of pulmonary hypertension also suggested pulmonary embolism but the resemblance was superficial. In most cases, the clinical setting suggested the possibility of infection, the onset of symptoms was more gradual, constitutional symptoms were more prominent, and dyspnoea less conspicuous. Furthermore, the degree of rise of the jugular venous pressure in these patients was less than would have been expected had pulmonary embolism been the sole cause of systemic hypotension. Hence, clinical distinction from pulmonary embolism was possible and pulmonary angiography was not required in any of these patients.

Angiography and the site and severity of obstruction The angiographic findings which are typical of major pulmonary embolism have been adequately described (Simon and Sasahara, 1965). Our interest has been rather to correlate the degree of pulmonary vascular occlusion in the pulmonary angiogram with the clinical and haemodynamic evidence of the severity of embolism. It has long been known that 50 per cent or more of the pulmonary vascular bed must be occluded either in man or in animals before pulmonary hypertension results (Brofman et al., 1957; Gibbon and Churchill, 1936; Ebert et al., 1967). On this basis, our observations suggest that the obstruction to pulmonary blood flow in major pulmonary embolism is due to a mechanical obstruction in the proximal pulmonary arteries, as has been widely assumed. Thus, the degree of occlusion of the proximal pulmonary arteries was sufficient to account for the presence of pulmonary hyper- 
tension in all but 3 of our patients who had pulmonary vascular occlusion estimated to be less than 50 per cent. One of these exceptions had a saddle embolus which could have been causing more severe obstruction to the right or left main branch of the pulmonary artery than was evident in the pulmonary angiogram. However, there were 2 other patients in whom the extent of pulmonary vascular occlusion was 25 and 30 per cent, respectively. Since a small proportion of patients who have died of major pulmonary embolism have been found at necropsy to have severe occlusion confined to more distal vessels (Sevitt and Gallagher, I96r; Donaldson et al., I963) and since obstruction of vessels distal to tertiary branches of the pulmonary artery cannot be adequately assessed by pulmonary angiography (Dalen et al., 1966), it seems likely that more distal vascular obstruction was the cause of the apparent discrepancy between pulmonary angiogram and haemodynamic findings. Another piece of indirect evidence which suggested that most obstruction is proximal and mechanical in nature is the fact that the most severe degree of pulmonary vascular obstruction shown in our patients was 75 per cent, a figure that is in good agreement with the observation that anaesthetized cats (Gibbon and Churchill, 1936) and dogs (Ebert et al., 1967) can tolerate sequential occlusion of the lobar arteries until approximately 85 per cent of the pulmonary vascular bed has been occluded. We have therefore concluded that the pulmonary vascular occlusion in recent acute major pulmonary embolism is due to mechanical occlusion of large proximal pulmonary arteries which is clearly visible in the pulmonary angiogram in most patients, but due to occlusion of smaller and more distal arteries in a minority. However, the haemodynamic effects of isoprenaline infused into some of ' our patients suggested that there was a vasoconstrictive component to the pulmonary vascular obstruction even in the presence of severe occlusion of proximal vessels (MCDonald et al., 1968).

- Haemodynamics The severity of pulmonary hypertension in our patients was only moderate and did not accurately reflect the severity of pulmonary vascular obstruction, a finding that has been previously reported in experimental pulmonary embolism (Dalen et al., 1967). Thus, the severity of acute obstruc, tion to the right ventricle is reflected more by the extent of reduction in the stroke volume and cardiac output than by the severity of pulmonary hypertension, presumably because the chamber is thin walled and poorly adapted to sustain the high levels of right ventricular systolic pressure, which would be needed to sustain the stroke volume in the face of severe obstruction (Fineberg and Wiggers, 1936).

Investigations and management When oxygen has been administered and arterial hypoxia satisfactorily relieved, it is the ability of the right ventricle to produce an adequate stroke volume and cardiac output that determines whether or not a patient with major pulmonary embolism will survive. The stroke volume and cardiac output are therefore the best indicators of the severity of pulmonary vascular obstruction and of the ability of the right ventricle to overcome it. Other measurements that reflect the adequacy of the compensation of the circulation as a whole are the systemic blood pressure, mixed venous oxygen saturation, arterial $p \mathrm{H}$, and the urinary output. The pulmonary angiograms provide an important estimate of the 'reserve capacity' of the pulmonary circulation to tolerate further obstruction, since the presence of severe pulmonary vascular occlusion, even in the absence of severe haemodynamic changes, indicates that recurrent pulmonary embolism will probably be poorly tolerated. The pulmonary angiogram also reveals the distribution of the embolic material and its accessibility for surgical removal. The majority of patients in the present study had obstruction in the terminal portions of the main branches of the pulmonary artery, or of multiple lobar branches. This seems to represent a stable clinical situation. On the other hand, there is evidence that saddle pulmonary embolism may be an unstable situation carrying the threat of syncope or cardiac arrest due to a sudden exacerbation of pulmonary vascular obstruction (McDonald et al., 1970).

\section{References}

Barritt, D. W., and Jordan, S. C. (1960). Anticoagulant drugs in the treatment of pulmonary embolism: a controlled trial. Lancet, I, 1309.

Brofman, B. L., Charms, B. L., Kohn, P. M., Elder, J., Newman, R., and Rizika, M. (I957). Unilateral pulmonary artery occlusion in man, control studies. fournal of Thoracic Surgery, 34, 206.

Burdine, J. A., and Wallace, J. M. (1965). Pulsus paradoxus and Kussmaul's sign in massive pulmonary embolism. American fournal of Cardiology, 15, 413.

Cooley, D. A., and Beall, A. C., Jr. (I968). Embolectomy for acute massive pulmonary embolism. Surgery, Gynecology and Obstetrics, 126, 805.

Cross, F. S., and Mowlem, A. (1967). A survey of the current status of pulmonary embolectomy for massive pulmonary embolism. Circulation, 35 and 36, Suppl. I, 86. 
Dalen, J. E., Haynes, F. W., Hoppin, F. G., Jr., Evans, G. L., Bhardwaj, P., and Dexter, L. (1967). Cardiovascular responses to experimental pulmonary embolism. American fournal of Cardiology, 20, 3 .

Dalen, J. E., Mathur, V. S., Evans, H., Haynes, F. W., Pur-Shahriari, A. A., Stein, P. D., and Dexter, L. (1966). Pulmonary angiography in experimental pulmonary embolism. American Heart fournal, 72, 509.

Dexter, L., Dock, D. S., McGuire, L. B., Hyland, J. W., and Haynes, F. W. (1960). Pulmonary embolism. Medical Clinics of North America, 44, 1251 .

Donaldson, G. A., Williams, C., Scannell, J. G., and Shaw, R. S. (1963). A reappraisal of the application of the Trendelenberg operation to massive fatal embolism. New England fournal of Medicine, 268, I7I.

Ebert, P. A., Allgood, R. J., Jones, H. W., and Sabiston, D. C. (1967). Haemodynamics during pulmonary artery occlusion. Surgery, 62, 18.

Fineberg, M. H., and Wiggers, C. J. (1936). Compensation and failure of the right ventricle. American Heart fournal, II, 255.

Gibbon, J. H., Jr., and Churchill, E. D. (1936). The physiology of massive pulmonary embolism. An experimental study of the changes produced by obstruction to the flow of blood through the pulmonary artery and its lobar branches. Annals of Surgery, 104, 811.

Gray, F. D. (1966). Pulmonary Embolism, p. 87. Lea and Febiger, Philadelphia; Kimpton, London.

Gurewich, V., Sasahara, A. A., and Stein, M. (1965). Pulmonary embolism, bronchoconstriction and response to heparin. In Pulmonary Embolic Disease, pp. 162-169. Ed. by A. A. Sasahara and M. Stein. Grune and Stratton, New York and London.

Hale, G., Cade, J., McDonald, I. G., and Hirsh, J. (1968). Lung function studies in acute severe pulmonary embolism. Australasian Annals of Medicine, 17,194 .

Hirsh, J., Hale, G. S., McDonald, I. G., McCarthy, R. A., and Pitt, A. (1968). Streptokinase therapy in acute major pulmonary embolism: effectiveness and problems. British Medical fournal, 4, 729.

Little, J. M., and Loewenthal, J. (1968). Factors contributing to a lowered mortality from pulmonary embolism. Surgery, Gynecology and Obstetrics, 127, 777.

Luisada, A. A., and Cardi, L. (1956). Acute pulmonary oedema, pathology, physiology and clinical management. Circulation, 13, I13.

McDonald, I. G., Hirsh, J., and Hale, G. S. (I97I). Early rate of resolution of major pulmonary embolism; a study of angiographic and haemodynamic changes in the first 24 to 48 hours. British Heart fournal, 33, 432.

McDonald, I. G., Hirsh, J., Hale, G. S., Cade, J. F., and McCarthy, R. A. (1968). Isoproterenol in massive pulmonary embolism: haemodynamic and clinical effects. Medical fournal of Australia, 2, 201.
McDonald, I. G., Hirsh, J., Hale, G. S., Clarebrough, J. K., and Richardson, J. P. (I970). Saddle pulmonary embolism: a surgical emergency? Lancet, I, 269.

Marshall, R. (1965). Pulmonary Embolism Mechanism and Management, p. 25. Charles C. Thomas, Springfield, Illinois.

Miller, G. A. H., and Sutton, G. C. (1970). Acute massive pulmonary embolism; clinical and haemodynamic findings in 23 patients studied by cardiac catheterization and pulmonary arteriography. British Heart fournal, 32, 515.

Olazábal, F., Roman-Irizarry, L. A., Oms, J. D., Conde, L., and Marchand, E. J. (1968). Pulmonary emboli masquerading as asthma. New England fournal of Medicine, 278, 999.

Oram, S., and Davies, P. (1967). The electrocardiogram in cor pulmonale. Progress in Cardiovascular Diseases, 9, 341.

Sevitt, S., and Gallagher, N. (I96I). Venous thrombosis and pulmonary embolism: a clinico-pathological study in injured and burned patients. British fournal of Surgery, 48, 475.

Simon, M., and Sasahara, A. A. (1965). Observations on the angiographic changes in pulmonary thromboembolism. In Pulmonary Embolic Disease, pp. 214-224. Ed. by A. A. Sasahara and M. Stein. Grune and Stratton, New York and London.

Soloff, L. A., and Rodman, T. (1967). Acute pulmonary embolism, II-Clinical. American Heart fournal, 74, 829 .

Sutton, G. C., Honey, M., and Gibson, R. V. (1969). Clinical diagnosis of acute massive pulmonary embolism. Lancet, 1, 271.

Thomas, D. P. (1965). Treatment of pulmonary embolic disease. A critical review of some aspects of current therapy. New England fournal of Medicine, 273, 885 .

Tow, D. E., and Wagner, H. N. (1967). Recovery of pulmonary arterial blood flow in patients with pulmonary embolism. New England fournal of Medicine, 276, 1053 .

Walsh, P. N., Stengle, J. M., and Sherry, S. (1969). The urokinase-pulmonary embolism trial. Circulation, 39, 153.

Webster, J. R., Saadeh, G. B., Eggum, P. R., and Suker, J. R. (1966). Wheezing due to pulmonary embolism. Treatment with heparin. New England Fournal of Medicine, 274, 931.

Wechsler, B. M., Karlson, K. E., Summers, D. N., Krasnow, N., Garzon, A. A., and Chait, A. (1969). Pulmonary embolism: influence of cardiac haemodynamics and natural history on selection of patients for embolectomy and inferior vena cava ligation. Surgery, 65, 182.

Westermark, N. (1938). On the roentgen diagnosis of lung embolism. Acta Radiologica, 19, 357.

Requests for reprints to Dr. Ian G. McDonald, Cardiopulmonary Unit, St. Vincent's Hospital, Fitzroy 3065, Melbourne, Victoria, Australia. 